\title{
МЕТОД ОТСЕЧЕНИЯ ДЛЯ ЦЕЛОЧИСЛЕННЫХ ЗАДАЧ ЛИНЕЙНОГО ПРОГРАММИРОВАНИЯ С ДОПОЛНИТЕЛЬНЫМИ АЛЬТЕРНАТИВНЫМИ УСЛОВИЯМИ
}

В настоящей статье рассматривается полностью целочисленная задача линейного программирования с дополнительными альтернативными ограничениями. Для решения таких задач используется метод отсечения. Излагается прием для построения правильных отсечений. На основе третьего алгоритма Гомори строится конечный алгоритм.

\section{§ 1. Постановка задачи и вводные замечания}

Пусть в дальнейшем $B$ обозначает множество индексов базисных переменных, $N$ - множество индексов небазисных переменных и пусть $B \cup N=\{1,2, \ldots, n\}$.

Рассмотрим задачу: максимизировать функцию

$$
x_{0} \equiv a_{00}+\sum_{j \in N} a_{0 j}\left(-x_{j}\right)
$$

при ограничениях

$$
\begin{gathered}
x_{i} \equiv a_{i 0}+\sum_{j \in N} a_{i j}\left(-x_{j}\right) \geqslant 0, \quad i \in B, \\
x_{j} \equiv-\left(-x_{j}\right) \geqslant 0, \quad j \in N, \\
x_{j} \text { - целое при } j \in N
\end{gathered}
$$

и дополнительном условии, что имеетя одна или несколько групп ограничений вида $(m \geqslant n)$ :

$$
\begin{aligned}
& x_{m+1} \equiv a_{m+1,0}+\sum_{j \in N} a_{m+1, j}\left(-x_{j}\right) \geqslant 0 \\
& x_{m+p} \equiv a_{m+p, 0}+\sum_{j \in N} a_{m+p, j}\left(-x_{j}\right) \geqslant 0,
\end{aligned}
$$

где в каждой группе неравенств должно выполняться по меньшей мере одно (число неравенств в различных группах может быть неодинаковым).

Предполагаем, что все коэффициенты $a_{i j}$ в выражениях (1), (2) и (5) - целые числа. Тогда из соотношений (1), (2), (4) и (5) вытекает, что и переменные $x_{i}$, где $i \notin N$, - целые, так что рассматриваемая задача полностью целочисленная. Пусть общее число переменных в задаче (1)-(5) равняется $q$. 
П р и м е ч а и е. К рассматриваемым альтернативным условиям приводятся также $k$-кратные альтернативы, где требуется, чтобы среди $p$ неравенств типа (5) выполнялось $k$ неравенств $(k<p)$. Легко доказать, что для этого необходимо и достаточно, чтобы в каждом подмножестве из $p-(k-1)$ неравенств выполнялось по крайней мере одно.

Область, определенная условиями (2)-(3) и альтернативными условиями типа (5), является, вообще говоря, невыпуклой и может быть даже несвязной.

Как известно $\left[{ }^{1,2}\right]$, введением дополнительных булевых переменных задача математического программирования с альтернативными условиями может быть приведена к обычной задаче целочисленного программирования. Но это связано со значительным увеличением размерностей задачи. В $\left.{ }^{1}\right]$ предложен метод для решения полностью целочисленных задач линейного программирования с дополнительным условием. тто искомое решение входит в заданное множество $D$. В случае произвольного множества в [ $\left.{ }^{1}\right]$ применяются отсечения Данцига, что требует много шагов; в случае, когда $D$ выпукло или его дополнение $D^{\prime}$ до $E^{n}$ выпукло, строятся более сильные отсечения. В данном случае в качестве $D$ можно было бы взять множество, заданное альтернативными условиями. Но линейность альтернативных условий позволяет получить более простой метод построения отсечений. При этом выпуклость $D$ или $D^{\prime}$ не требуется.

\section{§ 2. Построение правильного отсечения}

Рассмотрим базисное решение системы * (2), (5):

$$
x_{j}=0 \text { при } j \in N, \quad x_{i}=a_{i 0} \quad \text { при } i \notin N,
$$

которое является, вообще говоря, псевдопланом рассматриваемой задачи. Предполагаем, что этот псевдоплан не удовлетворяет альтернативным условиям (5). Это значит, что

$$
a_{m+1,0}<0, \ldots, a_{m+p, 0}<0 .
$$

Но каждое допустим ое решение задачи (1)-(5) (и задачи (1)(3), (5)) удовлетворяет по крайней мере одному из неравенств (5), т. е. существует индекс $m+i \in\{m+1, \ldots, m+p\}$ такой, что при допустимом решении имеем

$$
\sum_{j \in N}\left(-a_{m+i, j}\right) x_{j} \geqslant-a_{m+i, 0}
$$

В силу (7) и (3) отсюда находим

$$
1 \leqslant \sum_{j \in N} \frac{a_{m+i, j}}{a_{m+i, 0}} x_{j} \leqslant \sum_{j \in N} \max _{1 \leqslant i \leqslant p} \frac{a_{m+i, j}}{a_{m+i, 0}} x_{j} .
$$

Обозначив

получим

$$
d_{j}=\max _{1 \leqslant i \leqslant p} \frac{a_{m+i, j}}{a_{m+i, 0}}
$$

$$
\sum_{j \in N} d_{j} x_{j} \geqslant 1
$$

или

$$
-1+\sum_{j \in N}\left(-d_{j}\right)\left(-x_{j}\right) \geqslant 0 .
$$

* Если альтернативных систем больше одной, то под (5) понимается множество всех таких систем. 
Неравенство (9) является правильным отсечением, так как по конструкции оно выполняется при каждом допустимом решении задачи ** (1) - (5), но не выполняется при псевдоплане, где $x_{j}=0, j \in N$. На основе неравенства (9) можно получить новое правильное отсечение с целыми коэффициентами

$$
[-1 / \lambda]+\sum_{j \in N}\left[-d_{j} / \lambda\right]\left(-x_{j}\right) \geqslant 0,
$$

где $\lambda$ - некоторое положительное число ([ $\left.{ }^{1}\right]$, с. 192).

\section{$\S 3$. Конечный алгоритм для решения поставленной задачи}

Рассмотрим задачу, поставленную в $§ 1$. Для $j=0$ и $j \in N$ обозначнм

и

$$
A_{j}=\left(a_{0 j}, a_{1 j}, \ldots, a_{n j}\right)^{\mathrm{T}}
$$

$$
\bar{A}_{j}=\left(a_{0 j}, \ldots, a_{n j}, a_{n+1, j}, \ldots, a_{m+1, j}, \ldots, a_{m+p, j}, \ldots, a_{q j}\right)^{\mathrm{T}},
$$

где $a_{i j}=0$ при $i \in N(i \neq j)$ и $a_{j j}=-1(j \in N)$, а знак «Т» обозначает транспонирование. Здесь $a_{n+1, j}, \ldots, a_{m+1, j}, \ldots, a_{m+p, j}, \ldots, a_{q j}-$ коэффициенты альтернативных условий типа (5) (если имеется только одна группа условий (5), то $n+1=m+1$ и $q=m+p)$.

Будем называть целочисленную таблицу

$$
A=\underbrace{\left(\bar{A}_{0}, \ldots, \bar{A}_{j}, \ldots\right)}_{1+|N| \text { столбцов }}, \quad j \in N,
$$

$l$-нормальной ([ $\left.\left.{ }^{1}\right]\right)$, если $A_{j}>0$ при всех $j \in N$, и допустимой, если $a_{i 0} \geqslant 0$ при $i \in B$ и среди чисел каждой группы $a_{m+1,0}, \ldots, a_{m+p, 0}$ имеется по крайней мере одно неотрицательное. Если таблица $A$ одновременно $l$-нормальна и допустима, то базисное решение (6) - оптимально.

Для дальнейшего изложения предположим, что исходная симплексная таблица $A l$-нормальна, целевая функция (1) ограничена снизу на множестве допустимых решений и известна некоторая нижняя граница целевой функции $a$ (последнюю можно определить, например, нахождением минимального значения функции (1) при условиях $(2)-(3))$. Тогда на основании третьего алгоритма Гомори $\left[{ }^{1,2}\right]$ с учетом модификации, предложенной в [ $\left.{ }^{1}\right]$, получается следующий алгоритм решения задачи (1)-(5).

1. Проверяем выполнимость условия $a_{00} \geqslant a$. При положительном ответе переходим к п. 2, при отрицательном - задача неразрешима.

2. Проверяем наличие среди $a_{i 0}, i \in B$, отрицательных. Если их нет, переходим к п. 3. Если имеются, выбираем первое из них, пусть это будет $a_{k 0}$, и проверяем, есть ли среди коэффициентов $a_{k j}$ отрицательные. Если нет- задача неразрешима. Если существуют, присваиваем $b_{j}:=a_{k j}(j=0$ и $j \in N)$ и переходим к п. 4.

3. Проверяем наличие групп альтернативных условий (5), для которых все свободные члены отрицательные. Если их нет, базисное решение (6) оптимально. Если есть, выбираем первую такую группу и строим отсечение $(9)$. Если все $-d_{j} \geqslant 0$, то альтернативные условия противоречивы и задача не имеет допустимых решений. Если имеется $-d_{j}<0$, присваиваем $b_{0}:=-1, b_{j}:=-d_{j}(j \in N)$ и переходим к ก. 4 .

** Неравенство (9) является правильным отсечением также для нецелочисленной задачи (1)-(3), (5), так как при его выводе условие (4) не используется.

*** Здесь $|N|$ обозначает мощность множества $N$, т. е. число его элементов. 
4. Определяем направляющий столбец $\bar{A}_{l}$ из условия

$$
A_{l}=\underset{b_{l}<0}{\operatorname{lex} \min } A_{j}
$$

и положительное число $\lambda$ из условия

где $\lambda_{1}$ вычисляется по формуле ****

$$
\lambda=\max \left\{-b_{l}, \lambda_{1}\right\},
$$

$$
\lambda_{1}=\min \left\{\mu: A_{j}+\left[b_{j} / \mu\right] A_{l}>0 \text { при всех } j \in N\right\} .
$$

Строим новое ограничение

где

$$
\begin{gathered}
x_{q+1}=a_{q+1,0}+\sum_{j \in N} a_{q+1, j}\left(-x_{j}\right), \\
x_{q+1} \geqslant 0, \quad x_{q+1}-\text { целое },
\end{gathered}
$$

$$
a_{q+1, j}=\left[b_{j} / \lambda\right] \quad(j=0 \text { и } j \in N) .
$$

Коэффициенты правой части соотношения (14) пишем снизу к таблице $A$, принимаем эту строку за направляющую и делаем симплексный шаг, т. е. переходим к новой таблице $A^{*}$ по формулам

$$
\bar{A}_{q+1}^{*}=\bar{A}_{l}, \quad \bar{A}_{j}^{*}=\bar{A}_{j}+a_{q+1, j} \bar{A}_{l} \quad(j \neq q+1) .
$$

При этом множества базисных и небазисных переменных и число переменных изменяются следующим образом:

$$
B^{*}=B \cup\{l\}, \quad N^{*}=N \cup\{q+1\} \backslash\{l\}, \quad q^{*}=q+1 .
$$

После симплексного шага переходим к п. 5.

5. Матрицу $A^{*}$, множества $B^{*}, N^{*}$ и числовую переменную $q^{*}$ обозначаем теперь через $A, B, N$ и $q$ соответственно и принимаем за текущие. Задача опять имеет вид (1)-(5). Переходим к п. 1.

Пр и м е ч а и е. При практическом применении алгоритма строка коэффициентов пострсенного отсечения после симплексного шага вычеркивается. Это гарантирует сохранение размеров симплексных таблиц.

В силу $a_{q+1,0}<0$ и $A_{l}>0$ из (16) следует, что всегда

$$
A_{0}^{*}=A_{0}-a_{q+1,0} A_{l}<A_{0} .
$$

Используя последнее неравенство, конечность изложенного алгоритма можно доказать почти так же, как конечность третьего алгоритма Гомори. Следуя рассуждениям $\left[{ }^{2}\right]$, приходим к выводу, что после конечного числа шагов вектор $A_{0}$ уже не изменяется и все $a_{i 0} \geqslant 0$ при $i \in B$. Легко доказать, что тогда и все альтернативные условия выполнены. В противном случае следовало бы построить отсечение (9) и после выполнения пп. 3 и 4 рассматриваемого алгоритма мы получили бы $A_{0}{ }^{*}<A_{0}$.

\section{§ 4. Численный пример}

В качестве примера рассмотрим задачу: максимизировать линейную форму

$$
x_{0} \equiv 5 x_{1}+6 x_{2}
$$

**** Определение $\lambda_{1}$ по условию (13) подробно описано в $\left[{ }^{1,2}\right]$, поэтому здесь на этом не останавливаемся. По формулам (12) и (13) получается наименьшее число $\lambda$, при котором направляющий элемент $a_{q+1, t}$ или $\left[b_{l} / \lambda\right]$ равняется -1 и новая симплексная таблица $\mathrm{A}^{*}$ является $l$-нормальной. 


\section{при условиях}

$$
\begin{aligned}
& x_{3} \equiv 10-2 x_{1}-x_{2} \geqslant 0, \\
& x_{1} \geqslant 0, \quad x_{2} \geqslant 0, \\
& x_{1}, x_{2}-\text { целые }
\end{aligned}
$$

и дополнительном условии, что среди следующих неравенств выполняются не менее двух:

$$
\begin{aligned}
& x_{4} \equiv 9-x_{1}-3 x_{2} \geqslant 0 \\
& x_{5} \equiv 21-14 x_{1}+4 x_{2} \geqslant 0, \\
& x_{6} \equiv 10+3 x_{1}-5 x_{2} \geqslant 0 .
\end{aligned}
$$

Заданное альтернативное условие равносильно требованию, чтобы в каждой паре неравенств (21) выполнялось по крайней мере одно (см. примечание в $\$ 1$ ).

\begin{tabular}{|c|c|c|c|}
\hline$x_{0}$ & 60 & 7 & 6 \\
\hline$x_{2}$ & $\begin{array}{r}0 \\
10 \\
0\end{array}$ & $\begin{array}{r}-1 \\
2 \\
0\end{array}$ & $\begin{array}{r}0 \\
1 \\
-1\end{array}$ \\
\hline$x$ & $\begin{array}{r}-21 \\
61 \\
-40\end{array}$ & $\begin{array}{r}-5 \\
22 \\
-13\end{array}$ & $\begin{array}{r}-3 \\
4 \\
-5\end{array}$ \\
\hline
\end{tabular}

Решение примера приведено в табл. $1-7$ (ведущий элемент отмечен звездочкой). Первоначальная симплексная табл. 1 не является $l$-нормальной, но она приводится к нужному виду после одного симп-

Таблица 1

\begin{tabular}{|r|r|rr|}
\cline { 2 - 4 }$x_{0}$ & 0 & -5 & -6 \\
\hline$x_{1}$ & 0 & -1 & 0 \\
$x_{2}$ & 0 & 0 & -1 \\
$x_{3}$ & 10 & 2 & $1 *$ \\
\hline$x_{4}$ & 9 & 1 & 3 \\
$x_{5}$ & 21 & 14 & -4 \\
$x_{6}$ & 10 & -3 & 5 \\
\hline
\end{tabular}

$x+$

$$
\begin{gathered}
\hline-4 \mid-1-1^{*} \\
\hline-1-\frac{13}{40}-\frac{1}{7} \\
\lambda=13 / 40
\end{gathered}
$$

\begin{tabular}{|c|c|c|c|}
\hline$\varepsilon_{0}$ & 33 & 1 & 5 \\
\hline$x_{1}$ & 3 & -1 & 1 \\
\hline . & $\begin{array}{l}3 \\
1\end{array}$ & 11 & $\begin{array}{l}0 \\
2\end{array}$ \\
\hline 8 & -3 & -2 & -1 \\
\hline 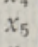 & -9 & 18 & -14 \\
\hline & 4 & -8 & \\
\hline
\end{tabular}

Таблица 5

\begin{tabular}{l|r|rr} 
& \multicolumn{1}{|c|}{1} & $-x_{9}$ & $-x_{7}$ \\
\cline { 2 - 4 }$x_{0}$ & 31 & 1 & 2 \\
\hline$x_{1}$ & 5 & -1 & 4 \\
$x_{2}$ & 1 & 1 & -3 \\
$x_{3}$ & -1 & 1 & -5 \\
\hline$x_{4}$ & 1 & -2 & 5 \\
$x_{5}$ & -45 & 18 & -68 \\
$x_{6}$ & 20 & -8 & 27 \\
\hline & & & \\
\hline & -1 & 0 & $-1^{*}$ \\
\hline
\end{tabular}

$\lambda=5$
Таблица 3

Таблица 4

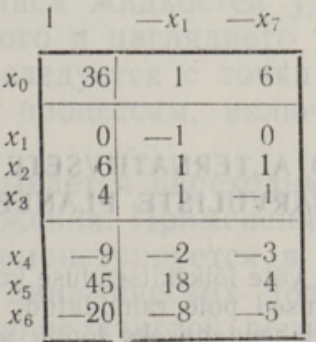

\begin{tabular}{|c|c|c|c|}
\hline$x_{0}$ & 28 & 1 & 3 \\
\hline $\begin{array}{l}x_{1} \\
x_{2} \\
x_{3}\end{array}$ & $\begin{array}{l}2 \\
3 \\
3\end{array}$ & $\begin{array}{r}-1 \\
1 \\
1\end{array}$ & $\begin{array}{r}3 \\
-2 \\
-4\end{array}$ \\
\hline $\begin{array}{l}x_{4} \\
x_{5} \\
x_{6}\end{array}$ & $\begin{array}{r}-2 \\
5 \\
1\end{array}$ & $\begin{array}{r}-2 \\
18 \\
-8\end{array}$ & $\begin{array}{r}3 \\
-50 \\
19\end{array}$ \\
\hline
\end{tabular}

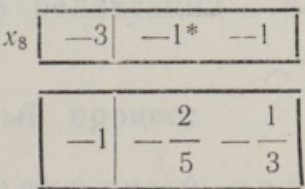

$\lambda=2 / 5$

$$
x_{9}-2-1 *-3
$$$$
-1-\frac{2}{3}-\frac{14}{9}
$$

$\lambda=2 / 3$

Таблица 7
Таблица 6

$$
\begin{array}{l|ll}
-1 & -\frac{8}{7} & \frac{5}{4} \\
\hline
\end{array}
$$

$\lambda=8 / 7$ 
лексного шага. Исходной для применения алгоритма служит табл. 2. Первая строка под табл. 2-6 - строка коэффициентов ограничения (14), вторая - строка коэффициентов отсечения (9), по которой строится отсечение (14) в табл. $2-4$ и 6 . Под таблицами также показано соответствующее значение $\lambda$. Из табл. 7 получается оптимальное решение примера: $x_{1}=2, x_{2}=3, x_{3}=3, x_{4}=-2, x_{5}=5, x_{6}=1$; максимальное значение целевой функции: $x_{0}=28$.

Как геометрически легко проверить, ни область, определенная альтернативными условиями (21), ни ее дополнение не являются выпуклыми. То же самое можно сказать об области, определенной условиями (18) - (19) вместе с альтернативными условиями (21), и об ее дополнении.

\section{Л И ТЕРАТ У РА}

1. Корбут А. А., Финкельштейн Ю. Ю., Дискретное программирование, М., 1969 , c. $44-51,166-197$.

2. Ху Т., Целочисленное программирование и потоки в сетях, М., 1974, с. 300-309. $323-326$.

Тартуский государственный университет

Поступила в редакцию 1/IX 1975

\section{KIVISTIK}

\section{LOIKEMEETOD ALTERNATIIVSETE LISAKITSENDUSTEGA LINEAARSETE TÄISARVULISTE PLANEERIMISULESANNETE JAOKS}

Esitatakse lihtne võte lõikekitsenduse konstrueerimiseks juhul, kui vaadeldava ülesande alternatiivsed tingimused pole rahuldatud. Gomory III algoritmi alusel konstrueeritakse püstitatud ülesande lahendamiseks lōplik algoritm. Selle rakendamist demonstreeritakse arvulise näitega.

\section{KIVISTIK}

\section{A CUTTING-PLANE METHOD FOR THE LINEAR INTEGER PROGRAMMING PROBLEM WITH ADDITIONAL ALTERNATIVE CONSTRAINTS}

The author presents a simple method for generating legitimate cuts in programming problems with additional alternative linear constraints. A finite algorithm, similar to Gomory's all-integer algorithm, is obtained by an «integrization» of these cuts. A numerical example is considered. 